\title{
A review of paediatric tuberculosis in Denmark: 10-year trend, 2000-2009
}

\author{
Camilla Ingrid Hatleberg ${ }^{1}$, Julie B. Prahl², Jeppe Nørgaard Rasmussen ${ }^{3}$, \\ Peter Henrik Andersen ${ }^{3}$, Stephanie Bjerrum ${ }^{4}$, Vibeke Østergaard Thomsen ${ }^{2,5}$ and \\ Isik Somuncu Johansen ${ }^{4}$
}

\begin{abstract}
Affiliations: 'Dept of Infectious Diseases, Copenhagen University Hospital, Hvidovre, ${ }^{2}$ The International Reference Laboratory of Mycobacteriology, Statens Serum Institut, Copenhagen, ${ }^{3}$ Dept of Infectious Disease Epidemiology, Statens Serum Institut, Copenhagen, ${ }^{4}$ Dept of Infectious Diseases, Odense University Hospital, Odense, and ${ }^{5}$ Hospital Services and Emergency Management, Danish Health and Medicines Authority, Copenhagen, Denmark.
\end{abstract}

Correspondence: I.S. Johansen, Dept of Infectious Diseases, Odense University Hospital, Sdr. Boulevard 29, 5000 Odense C, Denmark.

E-mail: isikadadlnet.dk

ABSTRACT Paediatric tuberculosis (TB) is a key indicator for recent transmission and presents a reservoir for the disease. We describe trends in epidemiology, microbiological characteristics and treatment outcome in Denmark between 2000 and 2009. Data were retrieved from the national TB surveillance system and the International Reference Laboratory of Mycobacteriology.

In total, $323 \mathrm{~TB}$ cases were reported in children aged $<15$ years, accounting for $7.6 \%$ of all notified cases in Denmark. The overall incidence rate of childhood TB declined from 4.1 per 100000 to 1.9 per 100000 in the study period. Immigrant children comprised $79.6 \%$ of all cases, with the highest incidence rate of 94.1 per 100000 children in 2001. In contrast to immigrant children, the majority of Danish children were aged $<5$ years and had a known exposure to TB. Pulmonary TB was the commonest presentation. Only half of the cases were culture confirmed.

We observed an overall decreasing trend in the child to adult notification ratio, but a slight increase in the ratio when calculated specifically for ethnic Danes.

Childhood TB needs continuous attention with a special focus on risk groups. Emphasis on improving early TB case detection, contact tracing and further implementation of preventive treatment is necessary.

@ERSpublications

Childhood TB needs continuous attention with a special focus on risk groups http://ow.ly/o36u6

\footnotetext{
For editorial comments see page 678 .

Received: April 032013 | Accepted after revision: June 282013 | First published online: Aug 292013

Conflict of interest: Disclosures can be found alongside the online version of this article at www.erj.ersjournals.com 


\section{Introduction}

In 2010, there were 8.8 million tuberculosis (TB) cases worldwide, equalling a global incidence rate of 128 per 100000 population. Between $9.6 \%$ and $11 \%$ of cases occurred in children aged $<15$ years, with the majority of cases seen in high TB burden countries [1]. The exact extent of childhood TB is unknown due to the lack of a standard case definition, the wide spectrum of disease and inherent diagnostic difficulties [2]. Furthermore, until recently, the only cases reported with age disaggregation were smear-positive cases in developing countries, while for smear-negative and extrapulmonary TB, age disaggregation was not provided, so it is impossible to determine the real burden of childhood TB at the global level [3].

Many western European countries have registered an increasing incidence of TB amongst both adults and children over the past two decades [4-7]. This is mainly driven by immigration from high-incidence areas. In most countries of western Europe, childhood $\mathrm{TB}$ constitutes $4-7 \%$ of all $\mathrm{TB}$ cases and the highest incidence rate is found among immigrants, with $>40$ cases per 100000 immigrant children $[4,5]$.

Denmark has a low overall TB incidence rate of 6.8 per 100000 with a total of 380 notified cases in 2011 [8]. However, the incidence rate of TB has fluctuated during the past decades. In 2000 there was a peak of 548 new cases resulting in an incidence rate of 10.3 per 100000 population, due to an increasing number of immigrants arriving from high-incidence countries [9]. Since 2002, a slight decrease in the number of notified cases of TB has been observed paralleled with a decrease in immigration [10].

In the period between 1990 and 1999, a total of 349 children were notified for TB in Denmark and 78\% of these were immigrants. The TB incidence rate increased from 2.0 per 100000 children in 1990 to 5.3 per 100000 in 1999. The highest incidence rate was observed amongst immigrant children with an average of 46 annual cases per 100000 in the study period [11].

Emphasis on improvement of early TB case detection is required as childhood TB is a key indicator for recent transmission in society and because children are prone to severe manifestations of the disease and increased mortality. The European Centre for Disease Prevention and Control (ECDC) now recommends that each country should monitor trends in the ratio of the notification rate in children to that in adults as an indicator of case-finding and effective treatment in the country [12].

The scope of this national study was to describe the recent TB epidemiology, clinical and microbiological characteristics, and treatment outcomes in children aged $<15$ years in Denmark over a 10-year period.

\section{Material and methods}

\section{Study population}

The Department of Infectious Disease Epidemiology at Statens Serum Institut (Copenhagen, Denmark), which hosts the national TB surveillance system in Denmark, provided data on all notified TB cases aged $<15$ years between January 2000 and December 2009. For analysis, we included data on demographics, clinical manifestation, disease verification, source of infection, risk factors and treatment outcome. Nonhousehold exposure was defined as TB exposure that took place outside the house, mostly involving other family members and adults at day care centres. All microbiological results from specimens from the notified cases were retrieved from the International Reference Laboratory of Mycobacteriology (Statens Serum Institut). Smears and culture were carried out on all samples, and a nucleic acid amplification (NAA) test was performed if requested. Up to three initial samples per case obtained during the first 14 days of disease investigation were included in the calculations. The Danish Data Protection Agency granted permission for the study.

\section{Subdivision of the study population}

The study population was stratified according to immigrant status, which was defined as children born abroad or those born in Denmark for whom one or both parents had been born abroad, including in Greenland.

\section{Statistics}

General characteristics, stratified by immigrant status, are presented as percentage frequency, and differences between variables of interest amongst immigrants and Danes were compared using the Chisquared or Fisher's exact test. The results of the microbiological tests are also presented as percentage frequency. Overall incidence rates per 100000 children and incidence rate per 100000 children subdivided by age group (0-4, 5-9 and 10-14 years), sex and ethnicity were calculated using the mid-year estimates from Statistics Denmark (www.dst.dk/en.aspx). In addition, the ratio of case notification in children to that in adults was calculated as described in a recent ECDC publication [12]. The calculated incidence rates were plotted against calendar years and the mean trend in the ratio of case notification rate in children to that in 
adults was calculated and plotted for the entire observation period. All statistical tests were two-sided and the significance level was chosen as $\mathrm{p}<0.05$.

\section{Results}

In the study period, $323 \mathrm{~TB}$ cases in children aged $<15$ years were notified in Denmark. In total, $4224 \mathrm{~TB}$ cases were notified during this period and, thus, childhood TB accounted for $7.6 \%$ of the total TB cases.

\section{Demographic characteristics}

Ethnicity was known in all but three cases. In total, 66 (20.4\%) cases were Danes. 24 children originated from other European countries, mostly from the Balkans. Six children were of Greenlandic origin. Children of African origin comprised $57.9 \%(n=149)$ of the total number of TB cases in the immigrant group, with the majority of children originating from Somalia $(n=133)$. The second largest group of reported TB cases were from Asia with a total of 65 TB cases. Among these, children from Pakistan and Afghanistan made up the majority $(n=38)$ with a further 10 children of Middle Eastern origin. We had information on time of entrance into Denmark for 136 of the immigrant children. The median time from entering Denmark to diagnosis of TB in these cases was 3 years. 13 of the immigrant children were born in Denmark.

The general characteristics of childhood TB cases, stratified by immigrant status, are summarised in table 1. The cases amongst Danish children were characterised by being young (0-4 years: $46.9 \%$ ), predominantly male $(60.6 \%)$ and with a known non-household exposure to TB $(43.9 \%)$. By contrast, the majority of TB cases amongst immigrants were characterised by being older (10-14 years: $47.1 \%$ ), predominantly female $(57.2 \%)$ and with unknown or other exposure to TB (42.8\%).

\section{Disease characteristics}

Pulmonary TB was the commonest presentation of the disease in both Danish and immigrant children (table 1). A higher proportion of immigrant children had lymphatic TB ( $13.6 \%$ versus $4.5 \% ; \mathrm{p}=0.04)$ compared with Danish children, and spinal, gastrointestinal and cutaneous TB were only observed among immigrant children. However, tuberculous meningitis was more frequent in Danish children ( $7.3 \%$ versus $1.9 \% ; \mathrm{p}=0.02)$.

The TB diagnosis was verified by a positive culture in $161(49.8 \%)$ cases and there was no significant difference in the proportion of culture verification between the two groups $(p=0.17)$. In addition, we found an increase in positive culture with age (0-4 years: 43 out of 109, 5-9 years: 37 out of 80, 10-14 years: 81 out of $134 ; \mathrm{p}<0.01$ ). The diagnosis was more likely to be verified by tuberculin skin test (TST) in Danish children $(16.6 \%$ versus $8.2 \%$; $\mathrm{p}=0.04)$. A substantial number of cases were diagnosed by chest radiography in both groups (table 1).

\section{Incidence rate by age and sex}

The incidence rates of childhood TB given by age and sex are shown in figure 1. The median age of all children was 8 years. The highest incidence rate was 11.3 per 100000 amongst females aged 10-14 years in 2001. The incidence in this group of children decreased in the following years reaching 2.35 per 100000 in 2009 (fig. 1b).

\section{Trends in the ratio of the notification rate}

The 10-year trend in the ratio of TB case notifications in children to those in adults for all reported TB cases is presented in figure 2. The ratio decreased over the study period, although an increase was observed in 2005 and 2007 (fig. 2a). The highest notification rate amongst children was 4.8 per 100000 in 2001 and the lowest was 1.9 per 100000 in 2009 . When we studied the trend in the ratio of the case notification rate in Danish children to that in Danish adults, we observed a slightly increasing trend (fig. 2b). The notification rate for Danish children and adults was 0.5 and 4.8 per 100000 population, respectively, in 2000. In 2009, the notification rate for Danish children had increased to 0.6 per 100000 , while the rate for Danish adults had decreased to 2.6 per 100000 . In contrast, the ratio of the notification rates in immigrant children to immigrant adults decreased over the same period (fig. 2c). The highest notification rate for immigrant children was 94.1 per 100000 immigrant children in 2001. The rate decreased to 35.1 per 100000 in 2009. The incidence for adult immigrants decreased during the observational period (97.2 to 44.9 per 100000 ).

\section{Bacterial results}

Specimens were received for conventional mycobacterial diagnostics from 54 Danish children and 213 immigrant children. The results of microbiological tests from various specimens are shown in table 2. Of 90 sputum specimens, $28.8 \%$ were smear positive and $48.8 \%$ culture positive. 53 of the sputa were tested for the presence of Mycobacterium tuberculosis DNA by NAA and 27 (50.9\%) of these were positive. A total of 


\section{TABLE 1 Characteristics of tuberculosis cases in 323 children in Denmark}

\begin{tabular}{|c|c|c|c|}
\hline & Danes & Immigrants & Chi-squared \\
\hline Subjects & $66(20.4)$ & $257(79.6)$ & \\
\hline \multicolumn{4}{|l|}{ Sex } \\
\hline Male & $40(60.6)$ & $110(42.8)$ & 0.0097 \\
\hline Female & $26(39.4)$ & $147(57.2)$ & \\
\hline \multicolumn{4}{|l|}{ Age years } \\
\hline $0-4$ & 31 (46.9) & 78 (30.3) & 0.0003 \\
\hline $5-9$ & 22 (33.3) & $58(22.6)$ & \\
\hline $10-14$ & $13(19.7)$ & $121(47.1)$ & \\
\hline \multicolumn{4}{|l|}{ Risk factor } \\
\hline HIV/AIDS & & 1 & \\
\hline Household exposure & 26 (39.3) & 95 (36.9) & 0.7161 \\
\hline Non-household exposure & $29(43.9)$ & $51(19.8)$ & 0.0048 \\
\hline Unknown/other & $11(16.6)$ & $110(42.8)$ & 0.0046 \\
\hline \multicolumn{4}{|l|}{ Disease site } \\
\hline Pulmonary & $54(81.8)$ & $189(73.5)^{\#}$ & 0.1647 \\
\hline Milliary & $1(1.5)$ & $3(1.2)$ & 0.8197 \\
\hline Pleural & $2(3)$ & $8(3.1)$ & 0.9725 \\
\hline Lymphatic & $3(4.5)$ & $35(13.6)$ & 0.0413 \\
\hline Meningitis & $5(7.3)$ & 5 (1.9) & 0.0185 \\
\hline Spine & & $4(1.5)$ & \\
\hline \multicolumn{4}{|l|}{ Bone } \\
\hline Gastrointestinal & & $7(2.7)$ & \\
\hline \multicolumn{4}{|l|}{ Genito-urinary } \\
\hline \multicolumn{4}{|l|}{ Disseminated } \\
\hline Cutaneous & & $4(1.5)$ & \\
\hline Others & $1(1.5)$ & $2(0.7)$ & \\
\hline \multicolumn{4}{|l|}{ Disease verification } \\
\hline Culture positive & $28(42.4)$ & $133(51.7)$ & 0.1765 \\
\hline Smear positive & $1(1.5)$ & $1(0.4)$ & \\
\hline NAA positive & $3(4.5)$ & & \\
\hline Clinical symptoms & $3(4.5)$ & $4(1.5)$ & 0.1369 \\
\hline TST & $11(16.6)$ & $20(8.0)$ & 0.0393 \\
\hline IGRA & & $1(0.4)$ & \\
\hline Chest radiography & 20 (30.3) & $89(34.6)$ & 0.5072 \\
\hline Histology & & $7(2.7)$ & \\
\hline None available & & $2(0.7)$ & \\
\hline
\end{tabular}

Data are presented as $\mathrm{n}(\%)$, unless otherwise stated. NAA: nucleic acid amplification; TST: tuberculin skin test; IGRA: interferon- $\gamma$ release assay. \#: 17 immigrant children with pulmonary tuberculosis were also registered with extrapulmonary localisation (lymph nodes $(n=8)$, gastrointestinal $(n=3)$, spine ( $n=2)$, pleural $(n=1)$, meningitis $(n=1)$, genitourinary $(n=1)$ and skin $(n=1))$.

237 gastric fluids were received and $12.2 \%$ and $42.6 \%$ were smear and culture positive, respectively. M. tuberculosis was detected in $29 \%$ of gastric fluids tested by NAA. Acid-fast bacilli were detected by smear in $17.1 \%$ of tissue biopsies and in $62.8 \%$ M. tuberculosis was cultured. The best performance of smear, NAA and culture was found in pus material, where $47.6 \%, 75 \%$ and $90.4 \%$, respectively, were positive (table 2).

The time to culture positivity ranged from 5 to 65 days (median 16 days).

\section{Drug resistance}

Drug susceptibility test results were available for 159 of the cases. Resistance to any drug was found in 18 children (resistance rate 11.3\%). Mono-drug resistance to isoniazid was found in isolates from 13 children. Ethambutol monoresistance was found in isolates from two children. Pyrazinamide mono-drug resistance was found in an M. tuberculosis complex isolate from one child and in one Mycobacterium bovis isolate from another child. Resistance to rifampicin, isoniazid and ethambutol (multidrug resistance) was found in one isolate. This isolate was also resistant to rifabutin, but susceptible to amikacin, ofloxacin and capreomycin. Extensively drug-resistant TB has not yet been reported in Denmark. 

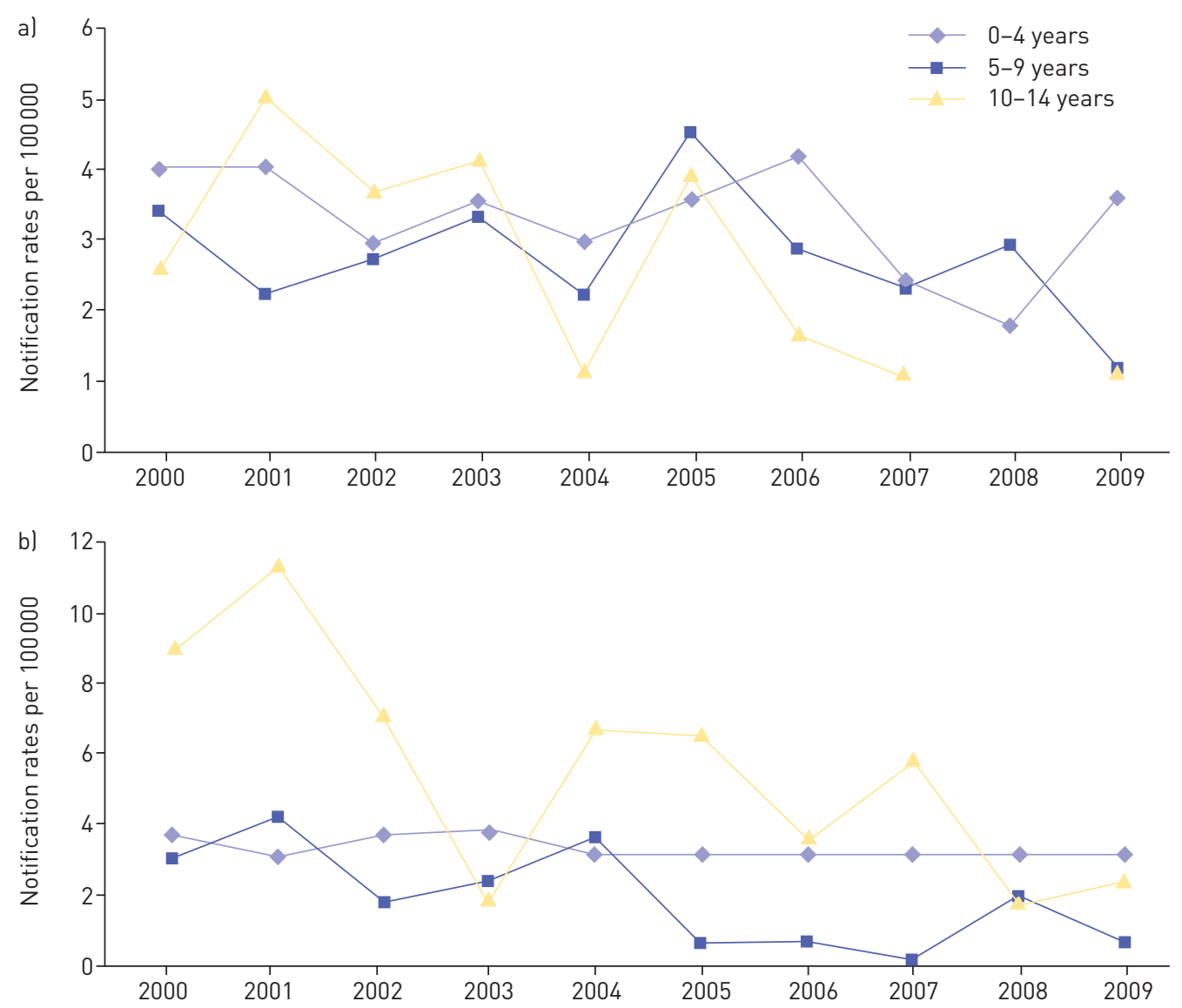

FIGURE 1 Incidence of childhood tuberculosis in a) males and b) females by age in Denmark between 2000 and 2009.

\section{Recurrence}

In total, three cases were registered with a recurrence during the study period. All were immigrant children and two of the cases had culture-verified TB. A patient was treated for TB spondylitis in 2001 and pulmonary TB in 2007, and drug-susceptible strains were cultured in both occurrences. Another patient was treated for drug-susceptible pulmonary TB in 2001 and 2005. The last case was treated for culture-negative pulmonary TB verified by chest radiography in 2006 and culture-negative lymphatic TB in 2007. The diagnosis was verified by a positive NAA test for M. tuberculosis in pus from cervical lymphadenitis.

\section{Treatment outcome}

Treatment outcome for both pulmonary and extrapulmonary TB has been reported by physicians since 2000 , but is not mandatory in Denmark. As the results from the year 2000 were highly incomplete they were excluded from analyses. In $226(79.1 \%)$ of the cases the outcome was "treatment completed" and the outcome "cured" was reported in $42(14.7 \%)$ cases, yielding an overall treatment success for the years 2001 to 2009 at $93.8 \%$. One child $(<1 \%)$ died (fig. 3).

\section{Discussion}

The epidemiology of TB in Denmark has changed over the past decade. Restrictions in the Danish immigration laws in the year 2000 resulted in a parallel decrease in immigration from high-incidence countries and a decline in the overall TB incidence rate from 10.3 to 6.0 per 100000 [9, 10]. In 2008, the ECDC introduced the Framework Action Plan to Fight TB in the European Union (EU) and in 2010 the supporting monitoring framework was published $[12,13]$. Four epidemiological indicators were proposed for monitoring and evaluating the quality of TB control. One of the indicators is childhood TB and the ECDC target is defined as a mean decline in the 10-year trend in the ratio of notification rates in children to that in adults. This trend reflects the change in the level of recent transmission and treatment efficiency in the country. We observed an overall decreasing trend in this ratio between 2000 and 2009, although fluctuations were seen. Interestingly, we found the opposite tendency when calculating this trend for Danish childhood cases versus Danish adult cases alone. The observed slightly increasing trend can be explained by 

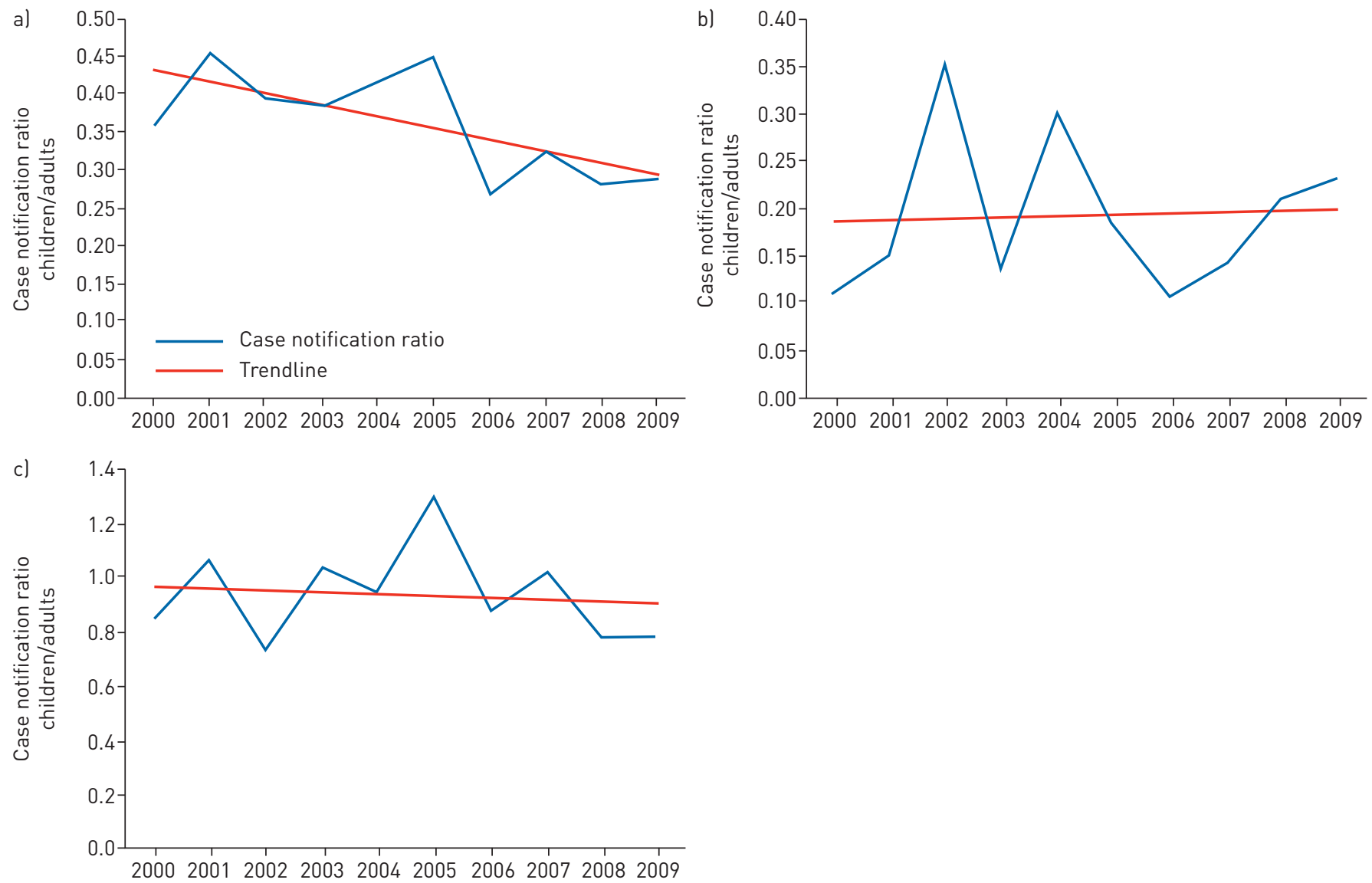

FIGURE 2 Trends in the ratio of notification rates in a) children to adults, b) Danish children to Danish adults and c) immigrant children to immigrant adults, between 2000 and 2009 in Denmark.

delays in case-finding among adult Danes. Although caution should be taken due to the low number of cases, this finding supports other observations that draw attention to the need for improved TB control among Danes; during the study period, the percentage of smear-positive adult pulmonary TB remained high at 60-70\% [14] and recently, KAMPER-JøRGENSEN et al. [15] have shown a high level of recent transmission, with a cluster frequency of $78 \%$ in a genotype epidemiology study. In particular, the outbreak strain Cluster 2 is found among young to middle-aged males living under difficult social conditions in the Danish capital Copenhagen, where the majority of cases were reported.

TABLE 2 Yield of Mycobacterium tuberculosis in smear, nucleic acid amplification (NAA) test and culture using various specimens in all notified cases for whom samples were obtained

Type of specimen

Specimens $\mathbf{n}$

Smear positive for AFB

Positive by NAA test for M. tuberculosis $\mathrm{n} / \mathrm{N}^{\#}(\%)$

Culture positive for M. tuberculosis

\begin{tabular}{|c|c|c|c|c|}
\hline Tracheal aspirates & 33 & $3(9)$ & $3 / 19(15.7)$ & $6(18.1)$ \\
\hline Gastric fluid & 237 & $29(12.2)$ & 45/155 (29) & $101(42.6)$ \\
\hline Pleura fluid & 10 & & $2 / 7(28.5)$ & $3(30)$ \\
\hline Cerebrospinal fluid & 16 & & $3 / 11(27.2)$ & $7(43.7)$ \\
\hline Pus & 21 & $10(47.6)$ & $9 / 12(75)$ & $19(90.4)$ \\
\hline
\end{tabular}

Data are presented as $\mathrm{n}(\%)$, unless otherwise stated. AFB: Acid-fast bacilli; BAL: bronchoalveolar lavage. ${ }^{\#}$ : N=number of NAA tests performed at clinician's request. 
FIGURE 3 Treatment outcomes for both pulmonary and extrapulmonary notified childhood tuberculosis between 2001 and 2009 in Denmark.

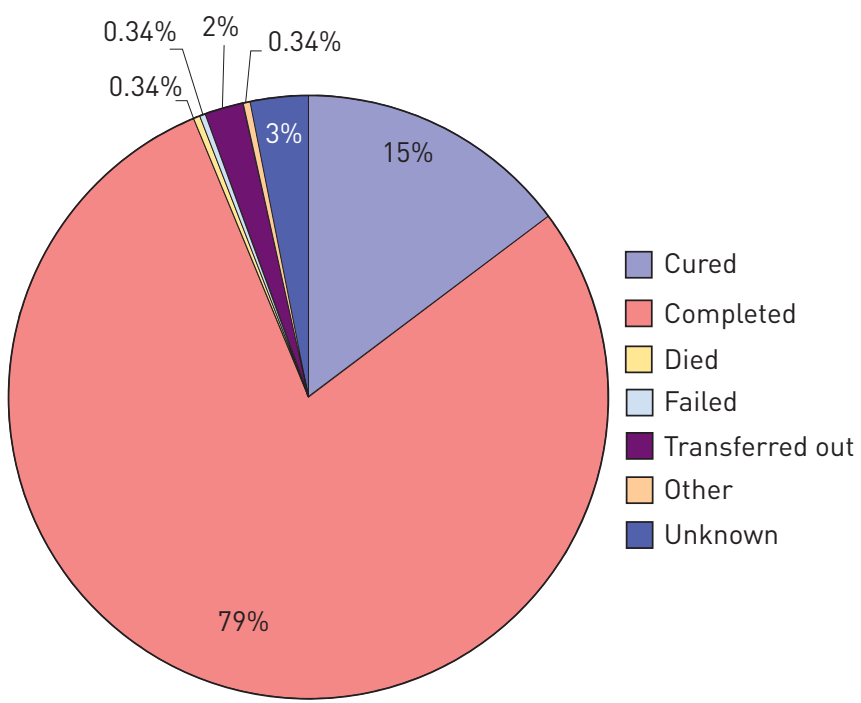

We found that the proportion of childhood TB remained high and accounted for $7.6 \%$ of the total TB burden in the observational period. This is comparable to the $8 \%$ observed in the previous decade [11]. This percentage stands at $4.3 \%$ in all EU/European Economic Area (EEA) countries [7]. As reported by a previous survey [11], children of foreign origin account for $80 \%$ of all childhood TB cases in Denmark. This is much higher than reported in the EU/EEA as a whole (29.2\%) [7]. In line with previous data from lowincidence settings $[4-6,16]$, this is an extremely high incidence rate for foreign-born children considering that most incident cases are found several or even many years after arrival in Denmark [17]. This is especially true for Somali children.

A recent study describing paediatric TB (aged <18 years) in Israel from 1999 to 2010 reported declining case rates during the study period [18]. Similar to Denmark, Israel is also a country with a low overall incidence of TB. The main findings of the Israeli study were a high proportion of childhood TB (8.4\%) and a very high incidence amongst children whose parents were born in Ethiopia (7.5 per 100000) and in the children of migrant workers (50-100 per 100 000), in line with our study. The majority of these children, who migrated to Israel, developed TB in the first year after arrival compared with a median period of 3 years after arrival in our study. However, there are important differences between Israel and Denmark with respect to $\mathrm{TB}$ case-finding. In Israel, initial screening for $\mathrm{TB}$ is routinely offered to all immigrants, whereas in Denmark TB screening is only offered to refugees and asylum seekers as they enter Denmark. The high case rates in immigrants originating from high $\mathrm{TB}$ incidence regions stresses the need for continuous attention towards this group and highlights the importance of early TB screening to diagnose all cases with latent and active disease, so that relevant treatment can be started as soon as possible.

The sex distribution of TB is well described amongst adults, with a male to female ratio of about two to one, which is found both in low- and high-incidence countries [19, 20]. However, the ratio is not well described for children. We found significant differences between Danish and immigrant children with respect to sex distribution. We do not have any explanation for this observation.

In general, children are at significantly higher risk of developing active disease after primary infection and age is one of the key parameters in this process. Infants and children aged $<2$ years were reported to be more vulnerable to developing the disseminated disease [21, 22]. Native Danish children aged 0-4 years were at significantly more risk of having TB than immigrant children in this age group. This feature was also observed in the EU/EEA region [7]. Conversely, for children aged 10-14 years, the immigrant group had a much higher risk of disease development than the Danish group.

The majority of the Danish children in this study had known contact with a TB patient. This is an important parameter to disclose since contact tracing with isoniazid preventive treatment may not have been optimal, as such measures would have lowered the risk of subsequent disease [23]. Furthermore, delays in diagnosing adult TB may have the result that children have developed the disease at the time of screening. Among immigrant children, TB exposure was unknown or reported to be "other" in $43 \%$ of the TB cases, which could reflect exposure before entering Denmark.

The majority of children in both groups suffered from pulmonary TB. Tuberculous meningitis was significantly more common amongst Danish children, which is explained by the lower age of TB cases in 
this group. Lymphatic TB was the second most common manifestation and was primarily reported amongst immigrant children. This is comparable to the national TB profile, where almost $90 \%$ of lymphatic TB occurred amongst immigrant patients $[14,24]$.

Ensuring a microbiologically verified diagnosis is still a major challenge in paediatric cases. Due to difficulties and inconvenience in sample collections, the percentage of specimen collection is usually low in the paediatric population. However, clinical specimens for microbiological tests were received in $82 \%$ of the current study population. Even higher percentages would be beneficial, but are probably difficult to achieve. Both acid-fast bacilli smear and culture were very insensitive, and even the performance of NAA was very low. The percentage of culture-confirmed TB was as low as 50\% in our paediatric population. However, this is comparable with previous reports from western Europe [4, 6, 7]. In 2009, only 19.2\% of paediatric TB cases were culture-confirmed in the EU/EEA, where optimal microbiological facilities are established [7]. The reason for the low sensitivity of bacteriological tests is primarily the paucibacillary nature of the disease in children. Submitting good-quality specimens is still important and encouraged in the new EU standards for TB care $[25,26]$.

The proportion of TB diagnoses based on a positive TST was significantly higher amongst Danish children who were not part of the bacille Calmette-Guérin vaccination programme terminated in 1984 and interpretation of a positive TST is probably easier in combination with clinical findings in the low-incidence population. This study is based on the epidemiological and microbiological data, which do not fully reflect the true and comprehensive process of assembling the diagnosis. Recently, published data based on information from case files found, that a combination of a positive TST, an abnormal chest radiograph and a history of exposure led to treatment commencement in $76 \%$ of cases [27]. There is a need for an improved diagnostic tool. The new lipoarabinomannan TB urine test may play an important role in the future. The test has shown promising results in diagnosing TB in severely immunocompromised HIV patients [28]. It could be of significant interest to investigate the performance of this test in a paediatric population.

Drug-resistant TB is not a major problem in Denmark, but the occurrence of resistance to first-line drugs in $11 \%$ of culture positive cases warrants close monitoring. Multidrug-resistant TB is rarely seen and extensively drug-resistant TB has not yet been reported $[29,30]$.

The overall treatment success of $94 \%$ in this study exceeded the target of $85 \%$ in the EU area [12]. Fortunately, childhood TB mortality was low, as only one child died. In order to improve surveillance of treatment outcome, we recommend mandatory reporting of treatment outcome, possibly combined with an electronic surveillance system.

\section{Conclusion}

Improvement in early case detection of adult $\mathrm{TB}$ cases is necessary to reduce transmission in the society, which in turn will ultimately reduce the number of childhood TB cases. Furthermore, an increased focus on contact tracing and preventive treatment is warranted. Finally, focus on groups at high risk for disease development may prove fruitful.

Paediatric TB represents failure to control transmission in the society and contributes significantly to the burden of disease as a reservoir; hence, continuous national and international commitment is necessary to ensure the highest standards of TB care and control in this highly vulnerable population [31].

\section{References}

1 World Health Organization. Global tuberculosis control 2011. Geneva, WHO press, 2011. www.who.int/tb/ publications/global_report/2011/en/

Nelson LJ, Wells CD. Global epidemiology of childhood tuberculosis. Int J Tuberc Lung Dis 2004; 8: 636-647.

Zar HJ, Hanslo D, Apolles P, et al. Induced sputum versus gastric lavage for microbiological confirmation of pulmonary tuberculosis in infants and young children: a prospective study. Lancet 2005; 365: 130-134.

Abubakar I, Laundy MT, French CE, et al. Epidemiology and treatment outcome of childhood tuberculosis in England and Wales: 1999-2006. Arch Dis Child 2008; 93: 1017-1021.

Ruwende JE, Sanchez-Padilla E, Maguire H, et al. Recent trends in tuberculosis in children in London. J Public Health (Oxf) 2011; 33: 175-181.

6 Nejat S, Buxbaum C, Eriksson M, et al. Pediatric tuberculosis in Stockholm: a mirror to the world. Pediatr Infect Dis J 2012; 31: 224-227.

7 Sandgren A, Hollo V, Quinten C, et al. Childhood tuberculosis in the European Union/European Economic Area, 2000 to 2009. Euro Surveill 2011; 16: 19825.

8 Statens Serum Institut. EPI-NEWS 2012; 50: Tuberculosis 2011, part 1. www.ssi.dk/English/News/EPI-NEWS/2012/ No\%2050\%20-\%202012.aspx Date last updated: December 12, 2012.

9 Statens Serum Institut. EPI-NEWS 2001; 43: Tuberculosis 2000, part 1. www.ssi.dk/English/News/EPI-NEWS/ / media/Indhold/EN\%20-\%20engelsk/EPI-NEWS/2001/pdf/EPI-NEWS\%20-\%202001\%20-\%20No\%2043.ashx Date last updated: October 24, 2001. 
10 Statens Serum Institut. EPI-NEWS 2010; 49: Tuberculosis 2009, part 1. www.ssi.dk/English/News/EPI-NEWS/2010/ No\%2049\%20-\%202010.aspx Date last updated: December 8, 2010.

11 Andersen PH, Thomsen VØ, Smith E. Tuberkulose blandt born i Danmark 1990-1999. [Tuberculosis among children in Denmark, 1990-1999]. Ugeskr Laeger 2001; 163: 6739-6742.

12 European Centre for Disease Prevention and Control. Progressing towards TB elimination. Stockholm, ECDC, 2010. www.ecdc.europa.eu/en/publications/Publications/101111_SPR_Progressing_towards_TB_elimination.pdf Date last updated: November, 2010.

13 European Centre for Disease Prevention and Control. Framework action plan to fight tuberculosis in the EU. Stockholm, ECDC, 2008. http://ecdc.europa.eu/en/publications/Publications/0803_SPR_TB_Action_plan.pdf Date last updated: February, 2008.

14 Statens Serum Institut. EPI-NEWS 2008; 51: Tuberculosis 2007, part II. www.ssi.dk/English/News/EPI-NEWS/ 2008.aspx Date last updated: December 17, 2008.

15 Kamper-Jørgensen Z, Andersen AB, Kok-Jensen A, et al. Clustered tuberculosis in a low-burden country: nationwide genotyping through 15 years. J Clin Microbiol 2012; 50: 2660-2667.

16 Winston CA, Menzies HJ. Pediatric and adolescent tuberculosis in the United States, 2008-2010. Pediatrics 2012; 130: e1425-1432.

17 Lillebaek T, Andersen AB, Dirksen A, et al. Persistent high incidence of tuberculosis in immigrants in a lowincidence country. Emerg Infect Dis 2002; 8: 679-684.

18 Mor Z, Cedar N, Pinsker G, et al. Childhood tuberculosis in Israel: epidemiological trends and treatment outcomes, 1999-2010. Eur Respir J 2013; 41: 1157-1162.

19 Thorson A, Diwan VK. Gender inequalities in tuberculosis: aspects of infection, notification rates, and compliance. Curr Opin Pulm Med 2001; 7: 165-169.

20 Martinez AN, Rhee JT, Small PM, et al. Sex differences in the epidemiology of tuberculosis in San Francisco. Int J Tuberc Lung Dis 2000; 4: 26-31.

21 Newton SM, Brent AJ, Anderson S, et al. Paediatric tuberculosis. Lancet Infect Dis 2008; 8: 498-510.

22 Drobac PC, Shin SS, Huamani P, et al. Risk factors for in-hospital mortality among children with tuberculosis: the 25-year experience in Peru. Pediatrics 2012; 130: e373-e379.

23 World Health Organization Stop TB Partnership Childhood TB Subgroup. Chapter 4: childhood contact screening and management. Int J Tuberc Lung Dis 2007; 11: 12-15.

24 Zhang X, Andersen AB, Lillebaek T, et al. Effect of sex, age, and race on the clinical presentation of tuberculosis: a 15-year population-based study. Am J Trop Med Hyg 2011; 85: 285-290.

25 Migliori GB, Sotgiu G, Blasi F, et al. Towards the development of EU/EEA Standards for Tuberculosis Care (ESTC). Eur Respir J 2011; 38: 493-495.

26 Migliori GB, Zellweger JP, Abubakar I, et al. European union standards for tuberculosis care. Eur Respir J 2012; 39: 807-819.

27 Rahman N, Pedersen KK, Rosenfeldt V, et al. Challenges in diagnosing tuberculosis in children. Dan Med J 2012; 59: A4463.

28 Lawn SD, Kerkhoff AD, Vogt M, et al. Diagnostic accuracy of a low-cost, urine antigen, point-of-care screening assay for HIV-associated pulmonary tuberculosis before antiretroviral therapy: a descriptive study. Lancet Infect Dis 2012; 12: 201-209.

29 European Centre for Disease Prevention and Control/WHO Regional Office for Europe. Tuberculosis surveillance and monitoring in Europe 2012. Stockholm, ECDC, 2012. www.ecdc.europa.eu/en/publications/Publications/1203Annual-TB-Report.pdf Date last updated: October 10, 2013.

30 Zignol M, Sismanidis C, Falzon D, et al. Multidrug-resistant tuberculosis in children: evidence from global surveillance. Eur Respir J 2013; 42: 701-707.

31 Sandgren A, Cuevas LE, Dara M, et al. Childhood tuberculosis: progress requires an advocacy strategy now. Eur Respir J 2012; 40: 294-297. 